\title{
A Monte Carlo Simulation of Radiation Damage of SiC and Nb Using JA-IPU Code
}

\author{
Nagendra Singh Raghaw ${ }^{2}$, Vinod Kumar ${ }^{1}$, Ambika Tundwal $^{1}$, Yury Korovin ${ }^{3}$ and Jindrich Adam ${ }^{4}$ \\ 1. University School of Basic and Applied Sciences, GGS Indraprastha University, New Delhi 110078, India \\ 2. HENPL (High Energy Nuclear Physics Laboratory) (ADS Program), University of Rajasthan, Jaipur 302004, India \\ 3. INPE (Institute for Nuclear Power Engineering), Kaluga Region, Obninsk 249020, Russia \\ 4. Laboratory for Nuclear Problem, Joint Institute of Nuclear Research, Dubna 141980, Russia
}

Received: July 19, 2015 / Accepted: September 01, 2015 / Published: November 30, 2015.

\begin{abstract}
MC (Monte Carlo) simulation code, JA-IPU is used to study radiation damage of SiC irradiated to spallation neutron and AmBe neutron spectra. The code is based on the major physical processes of radiation damage on incorporation of atomic collision cascade and limited to $10 \mathrm{MeV}$ neutron energy. A phenomenological relation for radiation swelling is also derived. Based on the calculation of swelling, DPA (displacement per atom), defect production efficiency and effective threshold energy, $E_{d}{ }_{d}^{\text {eff }}$ from the data of $\mathrm{MC}$ simulation, $\mathrm{SiC}$ is inferred to be a highly radiation resistant material when compared with $\mathrm{Nb}$ and $\mathrm{Ni}$ metals which are used in composition of several reactor steels. Experimental results of hill-hock density measured using AFM (atomic force microscopy), also confirm radiation resistant behavior of $\mathrm{SiC}$.
\end{abstract}

Key words: Monte-Carlo simulation, radiation resistant material, DPA, effective damage threshold energy.

\section{Introduction}

High energy spallation neutrons have important role to play in systems of energy like ADSS (accelerator driven sub critical) and fusion energy [1-3]. ADSS are assumed for both "nuclear energy" and "incineration of nuclear waste". In such systems, DPA (displacement per atom) is expected to be much higher compared to a critical system or a conventional reactor because energy of spallation neutrons is much higher than a critical reactor. This has generated a renewed interest in the studies of radiation damage [4-6] particularly for the development of new radiation resistant materials. At neutron energies up to several tens of $\mathrm{MeV}$ elastic collisions dominate the non-elastic collision.

In an elastic collision, the produced atomic recoil gives rise to a cascade of atomic collisions besides the primary cascade of incident particle. In case of

Corresponding author: Vinod Kumar, professor, research fields: ADS and transmutations of nuclear waste. E-mail: vkv1951@gmail.com. irradiation by neutrons a material is damaged both on the surface and in the bulk. Displacement of atoms may give rise to a number of physical effects like swelling, formation of voids, change in resistivity, induction of magnetism and change in the physical strength of a material. Monte Carlo simulation of atomic cascades, number of displaced atoms and their positions in the bulk, size of a voids, inter-atomic separation of the displaced atoms and ionization loses are very important to understand several new features arising due to an irradiation. In the JA-IPU code [7], we have introduced development of the atomic cascade and ionization loses which were not available so far in several codes used for the study of radiation damage. In the present study, we have carried out estimations of various quantities of radiation damage in case of $\mathrm{SiC}$ and $\mathrm{Nb}$ irradiated directly by the spallation neutrons produced in $660 \mathrm{MeV}$ proton $+\mathrm{Pb}$ collision and compared with the effects estimated in case of irradiation by a rather softer neutron spectrum of 


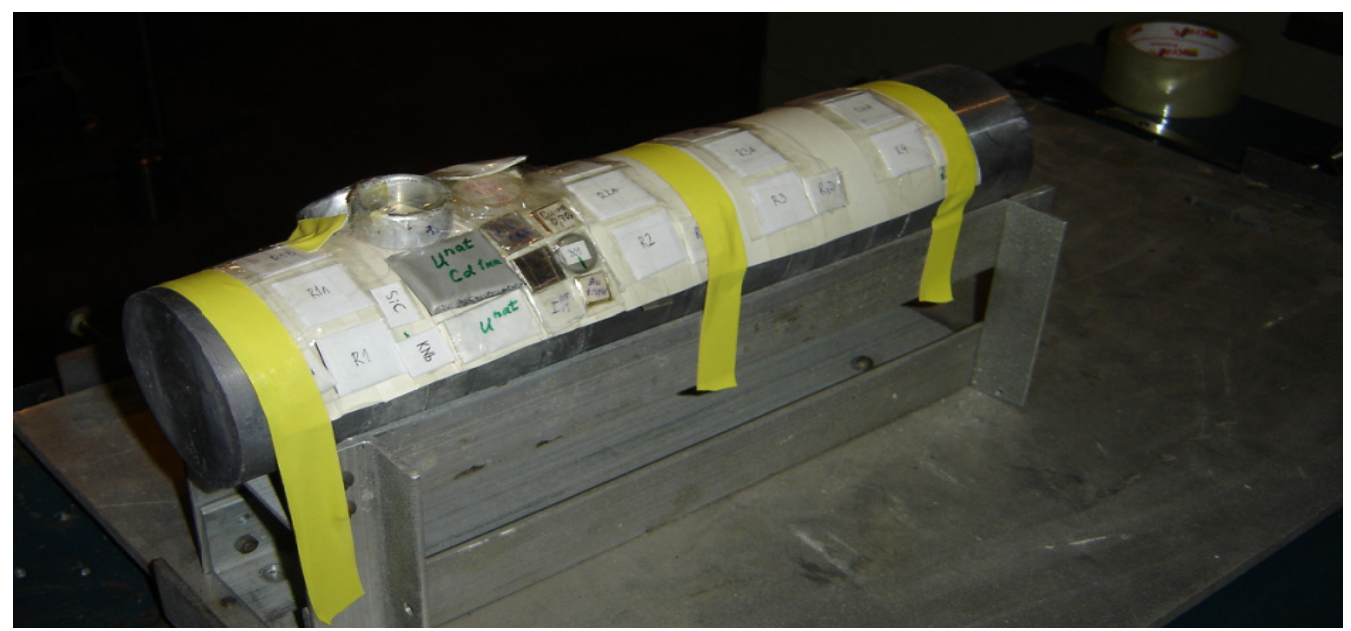

Fig. 1 Picture showing positions of activation samples on the upper half cylindrical surface of the lead spallation target. SiC and $\mathrm{Nb}$ samples assumed to be irradiated perpendicularly by the neutrons produced in the circular disc at $Z=6 \mathrm{~cm}$ from the left end of the target.

AmBe source. In the following Fig. 1, arrangement of irradiation of samples of $\mathrm{SiC}$ and $\mathrm{Nb}$ on the upper half cylindrical surface of lead spallation target of size $R \times L=4 \times 33 \mathrm{~cm}^{2}$ has been shown. Two samples are placed at $Z=6 \mathrm{~cm}$ from the left end where the $660 \mathrm{MeV}$ proton beam is supposed to strike the target.

It is highly important here to discuss the neutron fluence produced in the experimental system. For the estimation of intensity of proton beam, Al-monitor foils were put in the beam at $1.91 \mathrm{~m}$ before the spallation target and from the study of monitor reaction ${ }^{27} \mathrm{Al}(\mathrm{p}, 3 \mathrm{p} 1 \mathrm{n})^{24} \mathrm{Na}$ beam intensity is determined to be $2.66 \times 10^{10}$ protons $/ \mathrm{sec}$. The total irradiation time of samples after deducting beam pauses has been inferred to be 24,477 s. In the positions of the two samples, neutron fluence has been simulated using the CASCADE code ver. 2004 for $660 \mathrm{MeV}$ proton beam interacting with the lead cylinder. Results of simulation of the spallation neutrons per incident proton $(n / p)$ along the target length are given in Fig. 2. At the position of the samples, $Z=6 \mathrm{~cm}$ and in a width of $2 \mathrm{~cm}, n / p=2.22$, are emitted all around the cylindrical surface. This implies that, the neutron flux is $\sim 0.0443 \mathrm{n} / \mathrm{cm}^{2} / p$ and total neutron fluence on the samples is $2.88 \times 10^{13}$ neutrons $/ \mathrm{cm}^{2}$.

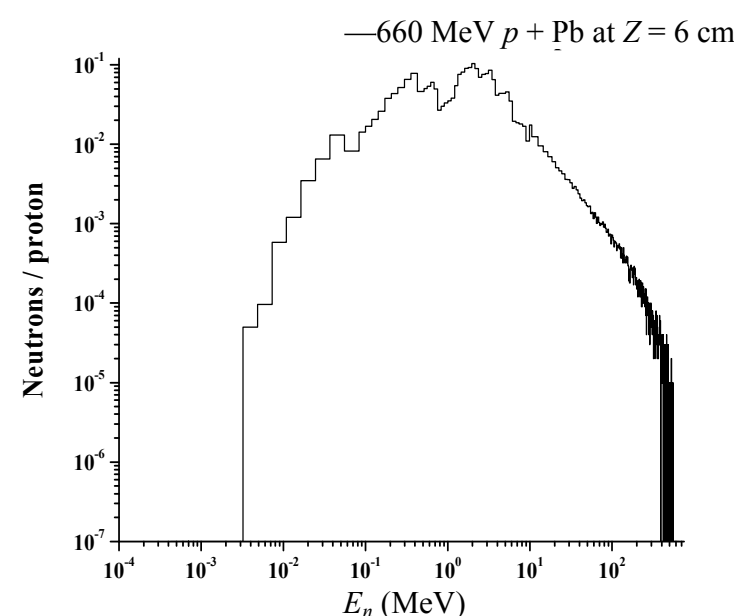

(a)

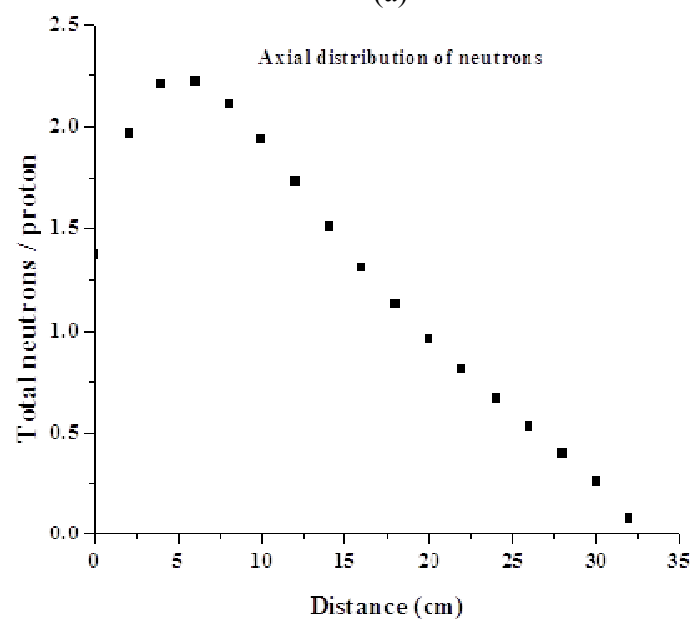

(b)

Fig. 2 (a) Neutron spectrum at the position of the samples, (b) results of Mote Carlo simulation from the CASCADE code given as variation of $n / p$ at different longitudinal $Z$-positions of the lead target in an interval of $2 \mathrm{~cm}$ length. 


\section{Monte Carlo Simulation of Radiation Damage}

JA-IPU code [7] has been used to simulate primary neutron + atom collisions and the secondary atomic cascade initiated by the displaced PKA (primary knocked out atom) of the collision. For this purpose, $n$ + atom interactions cross sections are taken from the ENDFVII.0 library and in the situation of non availability of cross sections in the library, cross sections are generated using TALYS code [8]. In case of atom + atom collision, cross sections are generated using the IOTA code [9] and in the following Figs. 3a-3c, plots of cross section of $\mathrm{C}+\mathrm{C}, \mathrm{Si}+\mathrm{C}$ and $\mathrm{Si}+\mathrm{Si}$ are shown as an example.

\subsection{Frenkel Pairs and Defect Efficiency}

In a collision when transferred kinetic energy is equal or greater than the threshold displacement energy of the atom, $E_{d}$ then a Frenkel pair is produced. The recoiled atom when gets energy larger than $E_{d}$ then it loses a part of its kinetic energy in ionization before it may further collide with another atom or it may stop or escape out of the material without a collision losing energy in ionization only. In calculations presented here, $T_{d a m}$ includes only the amount of energy that goes in producing displacements. For calculations, all recoiled atoms are assumed to be atomic nuclei for the purpose of estimation of the ionization. Thus, efficiency of defect production can be defined as follows:

$$
\eta=N_{d} / N_{N R T}
$$

where, $N_{d}$ is the number of simulated stable Frenkel pairs and $N_{N R T}$ is defined [10] as follows in terms of $T_{\text {dam }}$ :

$$
N_{N R T}=0.8 T_{\text {dam }} /\left(2 E_{d}\right)
$$

Primarily, a Frenkel pair is treated as a stable defect produced in a collision when transferred energy to an atom, $E>E_{d}$ and in the situation $E<E_{d}$, the energy may go in excitation of the atom. Both $T_{d a m}$ and $N_{d}$ are obtained from the Monte Carlo calculations. Results of Monte Carlo calculations obtained for the two samples irradiated by the spallation neutron spectrum using atom + atom collision cross sections are shown in Fig. 4 and compared with the results obtained for the $\mathrm{AmBe}$ neutron spectrum described in Ref. [7]. Detailed results of calculation for the spallation neutrons are given in Table 1 for the SiC. These results are based on $10^{10}$ histories of spallation neutrons. Results beyond neutron energy $5.06 \mathrm{MeV}$ have not been displayed due to the fact that no primary interaction is generated at higher energy due to small thickness of the sample. From the data displayed in Table 1, it may be noted that, value of simulated average damage energy per neutron per incident beam proton, $T_{d a m} /(n / p)$ is very small because of the fact that all incident neutrons do not produce interactions and further chances of secondary interactions also depend on the energy of the recoil produced in a primary collision. In case of the sample,

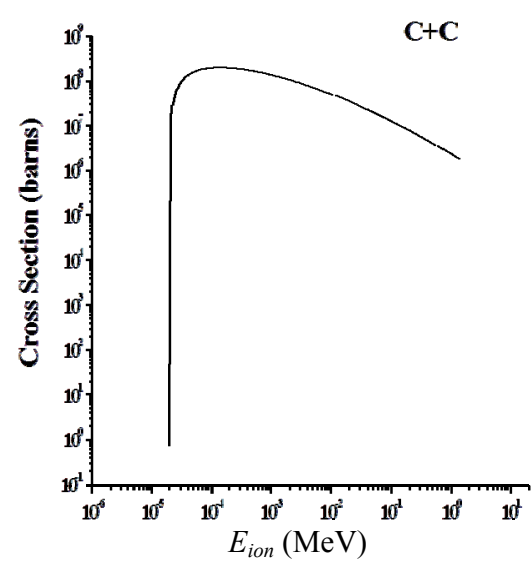

(a)

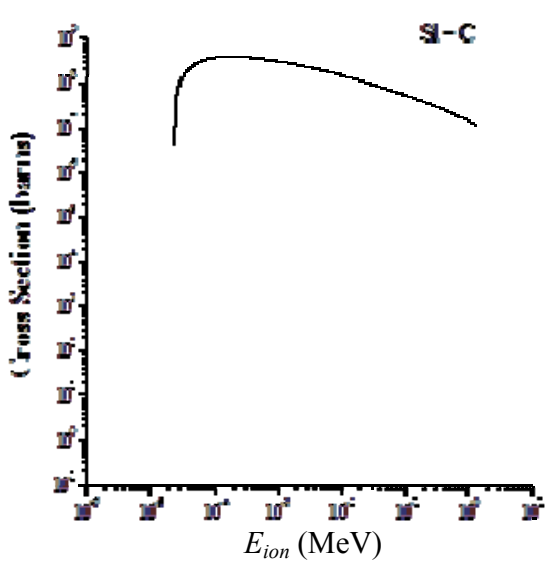

(b)

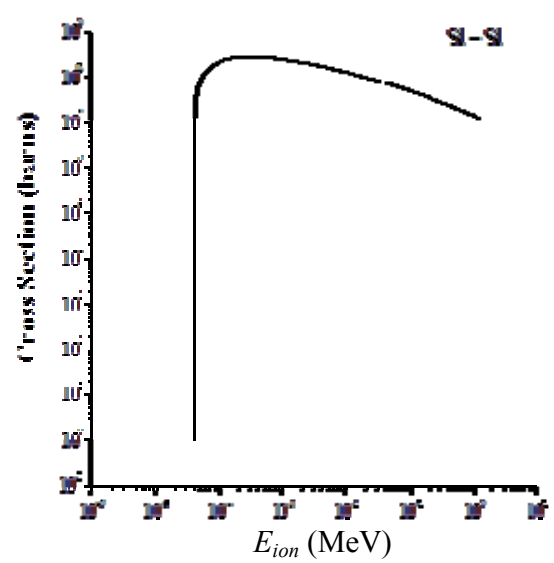

(c)

Fig. 3 (a) $\mathrm{C}+\mathrm{C}$ cross-section; (b) $\mathrm{Si}+\mathrm{C}$ cross-section; (c) $\mathrm{Si}+\mathrm{Si}$ cross-section. 


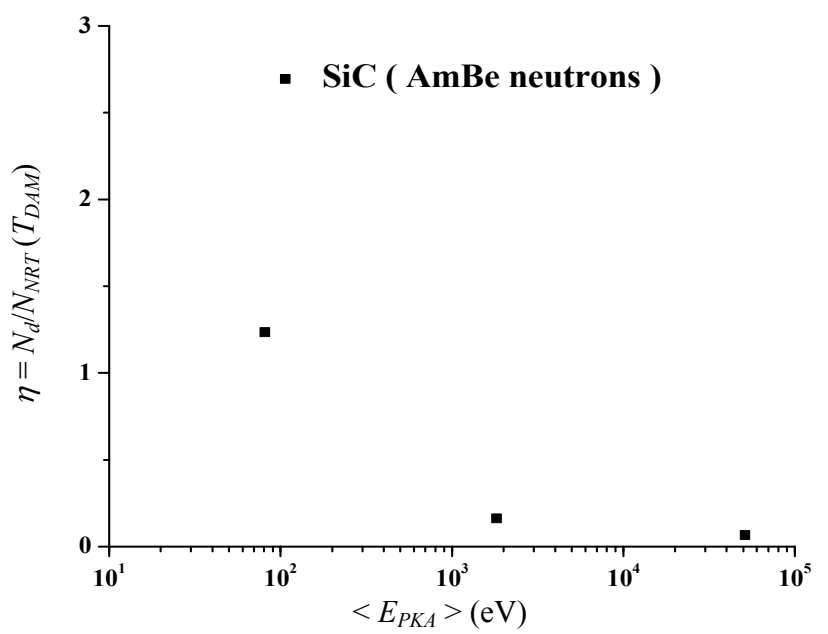

(a)

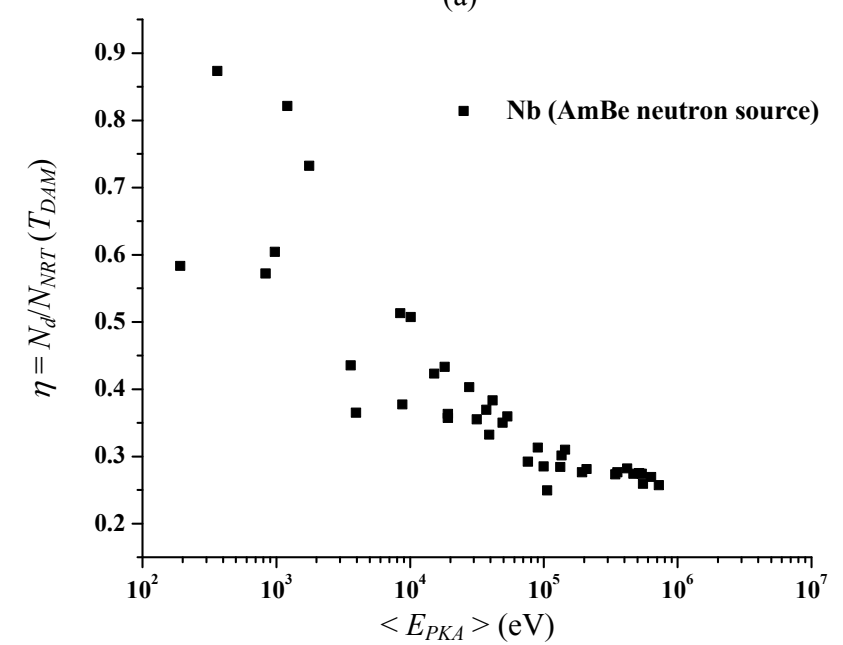

(c)

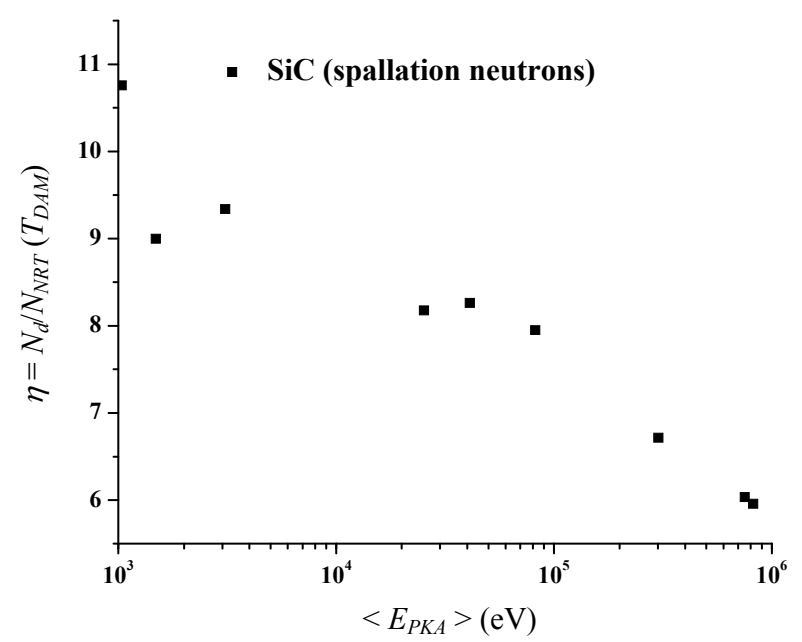

(b)

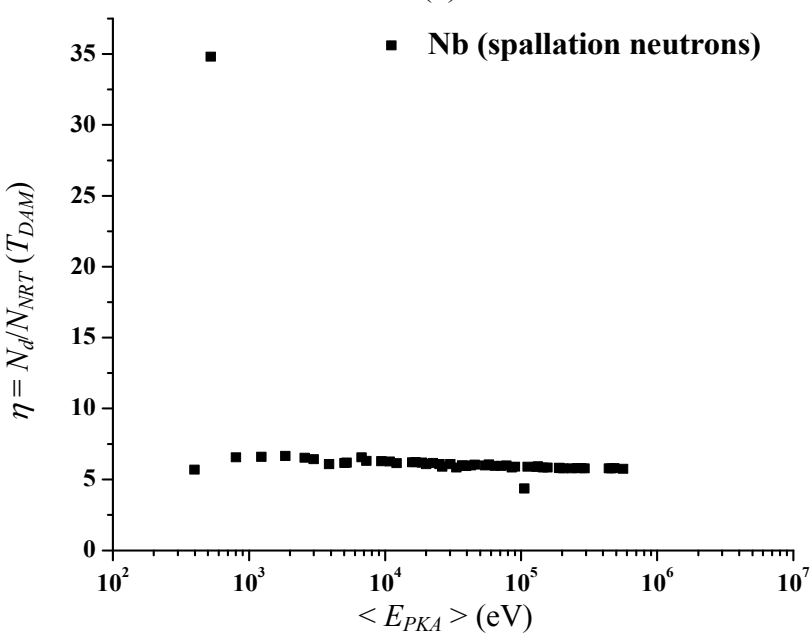

(d)

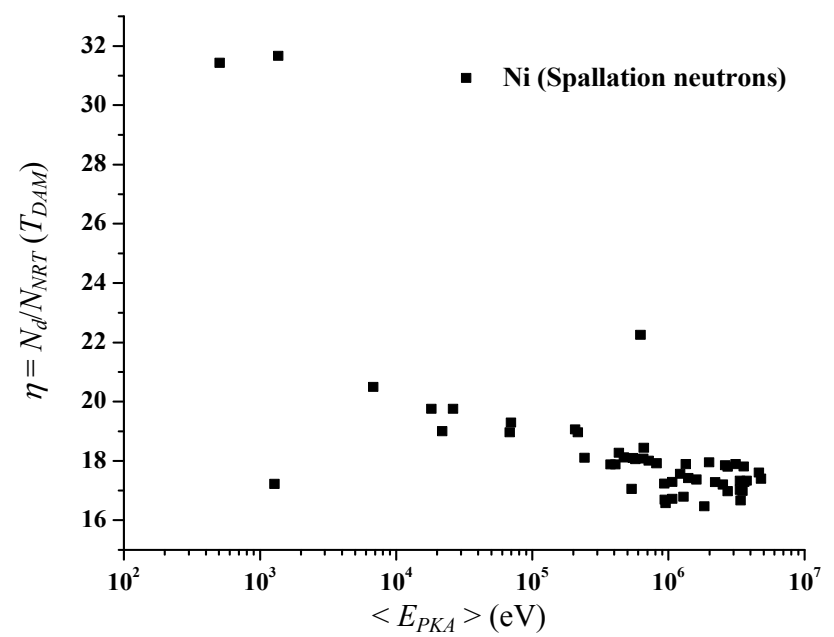

(e)

Fig. 4 (a) Defect production efficiency, $\eta$ as a function of average energy, $<E_{P K A}>(\mathrm{eV})$ for SiC irradiated to AmBe neutrons; (b) defect production efficiency, $\eta$ as a function of average energy, $<E_{P K A}>(\mathrm{eV})$ for $\mathrm{SiC}$ irradiated to spallation neutrons; (c) defect production efficiency, $\eta$ as a function of average energy, $<E_{P K A}>(\mathrm{eV})$ for Nb irradiated to AmBe neutrons; (d) defect production efficiency, $\eta$ as a function of average energy, $\left\langle E_{P K A}\right\rangle(\mathrm{eV})$ for Nb irradiated to spallation neutrons; (e) defect production efficiency, $\eta$ as a function of average energy, $<E_{P K A}>(\mathrm{eV})$ for $\mathrm{Ni}$ irradiated to spallation neutrons. 
Table 1 Results of $N_{d}\left(T_{d a m}\right), T_{d a m}, N_{N R T}\left(T_{d a m}\right)$ and $\eta$ for $\mathrm{SiC}$ calculated by generating histories of $10^{10}$ neutrons corresponding to the spallation neutron spectrum incident on the target.

\begin{tabular}{llllllll}
\hline$E_{n}(\mathrm{eV})$ & $(n / p)$ & $N_{d}\left(>E_{d}\right)$ & $N_{d}\left(<E_{d}\right)$ & $\begin{array}{l}T_{\text {dam }} /(n / p) \\
(\mathrm{eV})\end{array}$ & $\begin{array}{l}N_{N R T}\left(T_{\text {dam }}\right) \\
/(n / p)\end{array}$ & $\begin{array}{l}N_{d}\left(>E_{d}\right) / \\
(n / p)\end{array}$ & $\begin{array}{l}\eta=N_{d} / N_{N R T} \\
\left(T_{\text {dam }}\right)\end{array}$ \\
\hline $4.85 \times 10^{3}$ & $9.95 \times 10^{3}$ & $1.42 \times 10^{2}$ & $7.80 \times 10^{1}$ & $1.33 \times 10^{-1}$ & $1.33 \times 10^{-3}$ & $1.43 \times 10^{-2}$ & 10.7580 \\
$1.09 \times 10^{4}$ & $1.16 \times 10^{5}$ & $8.80 \times 10^{1}$ & $4.20 \times 10^{1}$ & $8.43 \times 10^{-3}$ & $8.43 \times 10^{-5}$ & $7.59 \times 10^{-4}$ & 8.9980 \\
$1.64 \times 10^{4}$ & $2.39 \times 10^{5}$ & $2.12 \times 10^{2}$ & $1.07 \times 10^{2}$ & $9.50 \times 10^{-3}$ & $9.450 \times 10^{-5}$ & $8.87 \times 10^{-4}$ & 9.3392 \\
$9.95 \times 10^{4}$ & $2.83 \times 10^{6}$ & $6.50 \times 10^{2}$ & $3.79 \times 10^{2}$ & $2.81 \times 10^{-3}$ & $2.81 \times 10^{-5}$ & $2.30 \times 10^{-4}$ & 8.1761 \\
$1.90 \times 10^{6}$ & $1.84 \times 10^{6}$ & $3.71 \times 10^{4}$ & $2.02 \times 10^{4}$ & $3.01 \times 10^{-1}$ & $3.01 \times 10^{-3}$ & $2.02 \times 10^{-2}$ & 6.7136 \\
$2.85 \times 10^{6}$ & $1.50 \times 10^{6}$ & $2.05 \times 10^{3}$ & $1.16 \times 10^{3}$ & $1.72 \times 10^{-2}$ & $1.72 \times 10^{-4}$ & $1.36 \times 10^{-3}$ & 7.9503 \\
$4.18 \times 10^{6}$ & $8.24 \times 10^{5}$ & $1.77 \times 10^{5}$ & $9.71 \times 10^{4}$ & $3.57 \times 10^{0}$ & $3.57 \times 10^{-2}$ & $2.15 \times 10^{-1}$ & 6.0333 \\
$4.60 \times 10^{6}$ & $8.68 \times 10^{5}$ & $1.06 \times 10^{3}$ & $6.09 \times 10^{2}$ & $1.48 \times 10^{-2}$ & $1.48 \times 10^{-4}$ & $1.23 \times 10^{-3}$ & 8.2609 \\
$5.06 \times 10^{6}$ & $8.74 \times 10^{5}$ & $1.94 \times 10^{4}$ & $1.042 \times 10^{4}$ & $3.72 \times 10^{-1}$ & $3.72 \times 10^{-3}$ & $2.22 \times 10^{-2}$ & 5.9573 \\
\hline
\end{tabular}

$\eta$ at small energies shows that simulated number $N_{d}(>$ $\left.E_{d}\right)$ is much higher than $N_{N R T}$ per incident $n / p$ and in case of higher neutron energies a part of PKA energy is not utilized for producing Frenkel pairs probably because PKA escapes out of the sample. As a result, $\eta$ declines to smaller value.

In the following Fig. 4, results of Monte Carlo calculations of defect production efficiency, $\eta$ as function of $<E_{P K A}>$ for $\mathrm{SiC}$ and $\mathrm{Nb}$ samples irradiated to both $\mathrm{AmBe}$ and spallation neutron spectra are displayed for comparison with results of $\mathrm{Ni}$ metal supposedly irradiated to spallation neutrons only because case of AmBe spectrum is already discussed [7]. It is revealed that, $\eta>1$ in case of $\mathrm{SiC}, \mathrm{Nb}$ and $\mathrm{Ni}$ irradiated to the spallation neutrons (detailed data of SiC is displayed in Table 1 also) and $\eta<1$ in case of the $\mathrm{SiC}$ and $\mathrm{Nb}$ irradiated to the $\mathrm{AmBe}$ neutron spectrum. Quantitatively, $\eta$ is 2-3 times higher in case of $\mathrm{Ni}$ than both $\mathrm{SiC}$ and $\mathrm{Nb}$ showing, $\mathrm{Ni}$ is more damaged than both $\mathrm{SiC}$ and $\mathrm{Nb}$ by the Frenkel pairs.

In Fig. 5, MC simulation results of $N_{d}$ and $T_{\text {dam }}$ are plotted for $\mathrm{SiC}, \mathrm{Nb}$ and $\mathrm{Ni}$ irradiated to the spallation neutrons along with $\mathrm{SiC}$ also irradiated to the $\mathrm{AmBe}$ moderated neutron spectrum [7]. It may be inferred that:

- In all the three cases of $\mathrm{SiC}, \mathrm{Ni}$ and $\mathrm{Nb}$ samples, there is linear dependence of growth of $N_{d}$ with $T_{d a m}$ per incident spallation and the AmBe neutron spectra. Values of slope " $k$ " are given in Table 2 for the three samples and values for $\mathrm{Ni}$ and $\mathrm{Nb}$ for irradiation by the moderated AmBe spectrum are taken from Ref. [7];

- In case of spallation neutrons, value of the slope, " $k$ " is much higher in case of $\mathrm{Ni}$ than $\mathrm{SiC}$ and $\mathrm{Nb}$ samples. It may be inferred that, to displace an atom of $\mathrm{SiC}$ or $\mathrm{Nb}$ is much harder than $\mathrm{Ni}$. This is further explored for the AmBe neutron spectrum where we can see that, the displacement is much more harder in case $\mathrm{SiC}$ and $\mathrm{Nb}$ than $\mathrm{Ni}$ compared to the case of spallation neutrons.

\subsection{Effects of Irradiation}

\subsubsection{Swelling}

Based on the linear relationship between $N_{d}$ and $T_{\text {dam }}$ shown in Fig. 5 and the data displayed in Table 2 for the two neutron spectra, a phenomenological approach is developed for estimation of swelling. According to this:

$$
\begin{aligned}
& \mathrm{d} N_{d} \propto \mathrm{d} T_{d a m} \\
& \mathrm{~d} N_{d}=k \mathrm{~d} T_{d a m}
\end{aligned}
$$

Constant, $k$ is spectrum dependent. On integration:

$$
\begin{aligned}
& \int \mathrm{d} N_{d}=k \int_{E_{d}}^{T_{d a m}} \mathrm{~d} T_{d a m} \\
& N_{d}=k\left(T_{d a m}-E_{d}\right)+C
\end{aligned}
$$

For $T_{\text {dam }}=0, N_{d}=0$ thus, $C=k E_{d}, \frac{N_{d}}{n}=$ $k\left(\frac{T_{d a m}}{n}-\frac{2 E_{d}}{n}\right)$ can be used for calculations per incident neutron.

Thus, the density of displaced atoms may be written as:

$$
n_{d}=\frac{N_{d}}{V}=k \frac{\left(T_{d a m}-2 E_{d}\right)}{V}
$$


Table 2 Sizes of $\mathrm{SiC}, \mathrm{Nb}$ and Ni samples and slope, $\mathrm{k}$ deduced from the data of $\mathrm{MC}$ (Monte Carlo) simulations.

\begin{tabular}{llll}
\hline & & \multicolumn{2}{l}{ Slope " $k$ " of $T_{\text {dam }}$ vs. $N_{d}$ plot } \\
\hline Samples & Dimensions of the samples & AmBe spectrum & Spallation spectrum \\
\hline $\mathrm{SiC}$ & $1 \times 1 \times 0.0314 \mathrm{~cm}^{3}$ & $0.00062 \pm 0.00003$ & $0.05992 \pm 0.00066$ \\
$\mathrm{Nb}$ & $2.4 \times 0.8 \times 0.024 \mathrm{~cm}^{3}$ & $0.00138 \pm 0.00001$ & $0.05762 \pm 0.00026$ \\
$\mathrm{Ni}$ & $2.5 \times 1.5 \times 0.002 \mathrm{~cm}^{3}$ & 0.875 & $0.16814 \pm 0.00045$ \\
\hline
\end{tabular}

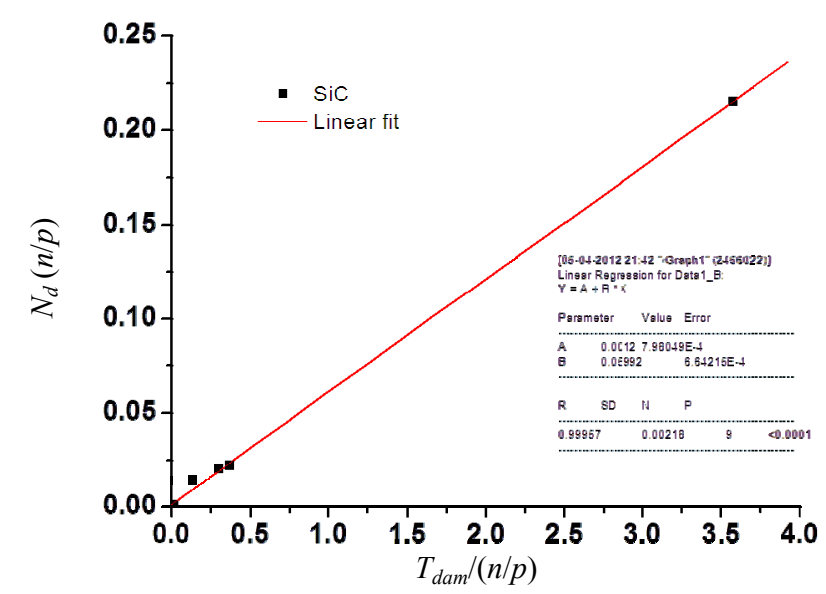

(a)

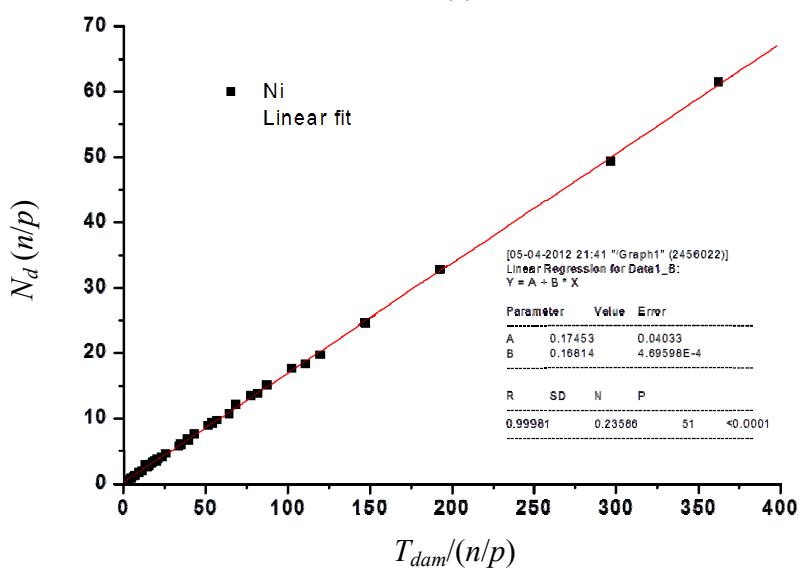

(c)

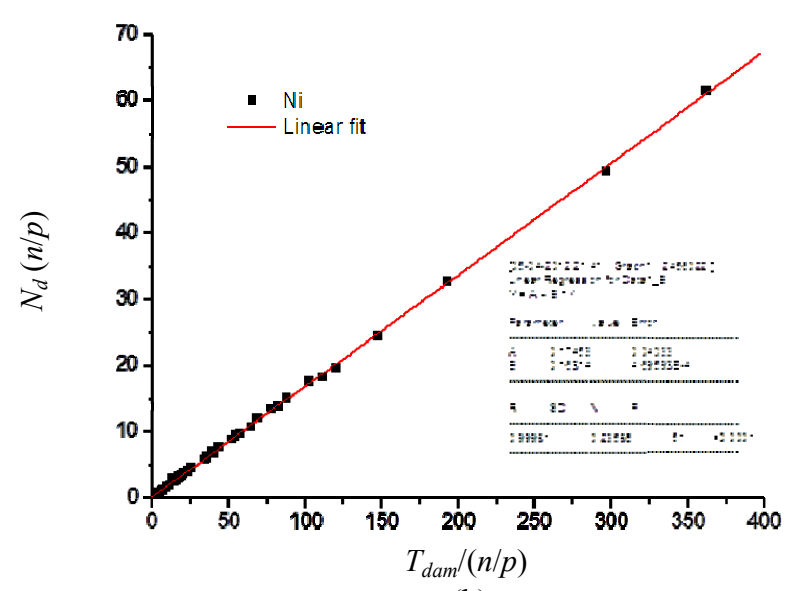

(b)

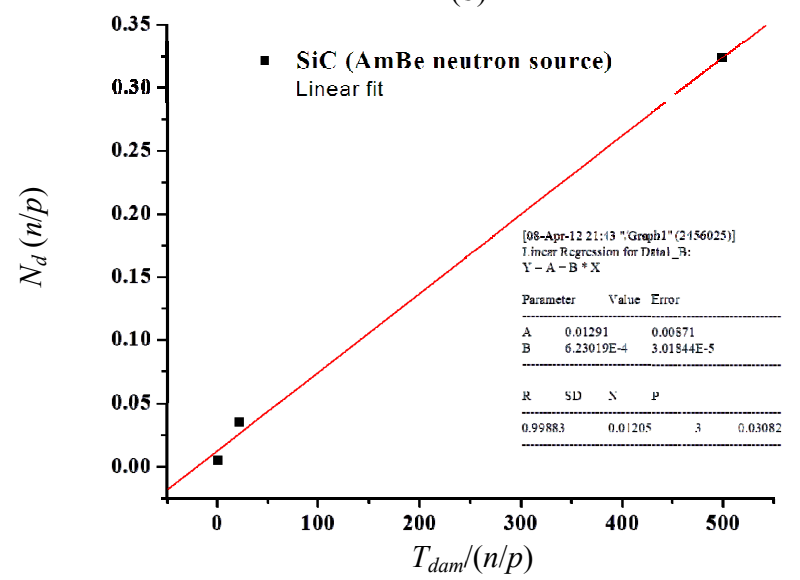

(d)

Fig. 5 (a) Number of displaced atoms, $N_{d}$ as function of damage energy, $T_{d a m}(\mathrm{eV})$ per neutron per proton $(n / p)$ of the spallation neutron spectrum in case of $\mathrm{SiC}$; (b) number of displaced atoms, $N_{d}$ as a function of damage energy, $T_{d a m}(\mathrm{eV})$ per neutron per proton $(n / p)$ of the spallation neutron spectrum in case of $\mathrm{Ni}$; (c) number of displaced atoms, $N_{d}$ as function of damage energy, $T_{\text {dam }}(\mathrm{eV})$ per neutron per proton $(n / p)$ of the AmBe moderated neutron spectrum in case of Nb; (d) number of displaced atoms, $N_{d}$ plotted as function of damage energy, $T_{d a m}(\mathrm{eV})$ per neutron per proton (n/p) of the AmBe moderated neutron spectrum in case of $\mathrm{SiC}$.

Thus,

$$
\begin{gathered}
n_{d}=n_{d}\left(T_{d a m}, V\right) \\
\mathrm{d} n_{d}=\left(\frac{\partial n_{d}}{\partial T_{\text {dam }}}\right) \mathrm{d} T_{d a m}+\left(\frac{\partial n_{d}}{\partial V}\right) \mathrm{d} V
\end{gathered}
$$

From Eq. (7)

$$
\begin{gathered}
\frac{\partial n_{d}}{\partial T_{d a m}}=\frac{k}{V} \\
\frac{\partial n_{d}}{\partial V}=-\frac{k}{V^{2}}\left(T_{d a m}-2 E_{d}\right) \mathrm{d} V
\end{gathered}
$$

Therefore,

$$
\mathrm{d} n_{d}=\left(\frac{k}{V}\right) \mathrm{d} T_{d a m}+\left(\frac{k}{V^{2}}\right)\left(T_{d a m}-2 E_{d}\right) \mathrm{d} V
$$

According to this, density of displaced atoms increases with the increase of $T_{d a m}$ and decrease of $\mathrm{d} V$.

Eq. (11) may also be rewritten as:

$$
\mathrm{d} n_{d}=\left(\frac{k}{V}\right) \mathrm{d} T_{d a m}-n_{d}\left(\frac{\mathrm{d} V}{V}\right)
$$


which leads to:

$$
\frac{\mathrm{d} V}{V}=\left(\frac{k}{V \cdot n_{d}}\right) \mathrm{d} T_{d a m}-\left(\frac{\mathrm{d} n_{d}}{n_{d}}\right)
$$

For low dose irradiation, last term may be considered negligible. Thus, the swelling, $\mathrm{d} V / V$ for a change of damage energy, $\mathrm{d} T_{\text {dam }}$ may be approximated by:

$$
S_{S W}=\frac{\mathrm{d} V}{V} \sim\left(\frac{k}{V \cdot n_{d}}\right) \mathrm{d} T_{d a m}=\left(\frac{k}{N_{d}}\right) \mathrm{d} T_{d a m}
$$

This shows that the swelling depends linearly on $\mathrm{d} T_{\text {dam }}$ and $k / N_{d}$ which in turn depends on the incident radiation spectrum.

Taking analogy with the process of thermal expansion, coefficient of swelling due to Frenkel pairs can be approximated from:

$$
\alpha_{S W}=\left(\frac{k}{V \cdot n_{d}}\right)
$$

For the purpose of analysis, displacement on the surface of an irradiated material can be accessed from the AFM (atomic force microscopy) analysis [7].

Spot views of AFM of the pristine and irradiated samples of $\mathrm{SiC}$ are given in Fig. 6. In Fig. 7, measured hill hock distribution is given. In the pristine sample hill-hock density comes out to be $\sim 12.79 \times 10^{10} / \mathrm{cm}^{2}$ and in case of irradiated sample, it is $\sim 23.39 \times$ $10^{11} / \mathrm{cm}^{2}$ showing that, on irradiation hill-hock density of the $\mathrm{SiC}$ sample increases only $\sim 18.3$ times. In case of $\mathrm{Ni}$ metal, [7] difference of hill-hock density in pristine and irradiated was very large because of the fact that $\mathrm{Ni}$ is a metal and is not a radiation resistant material like $\mathrm{SiC}$. In case of $\mathrm{Nb}$ sample, AFM analysis could not be possible due to its rough surface. Based on the data of volume of the sample and values of " $k$ " given in Table 2 and using Eq. (15), swelling coefficient, $\alpha_{s w}$ for $\mathrm{SiC}$ irradiated to the given spectrum of spallation neutrons, comes out to be $\sim 8.53 \mathrm{E}-13 \mathrm{~cm}^{-1} \mathrm{eV}^{-1}$. In case of $\mathrm{Ni}$ metal after irradiation by a relatively softer neutron spectrum of AmBe source [7], $\alpha_{s w}$ has been estimated to be $4.7 \mathrm{E}-10 \mathrm{~cm}^{-1} \mathrm{eV}^{-1}$. This is about 500 times higher than $\mathrm{SiC}$ irradiated to a higher energy neutron spectrum. This may be interpreted in favour of $\mathrm{SiC}$ to be a highly radiation resistant material with respect to its swelling behaviour. More experiments need to be performed in this direction where swelling and electrical resistance can be measured.

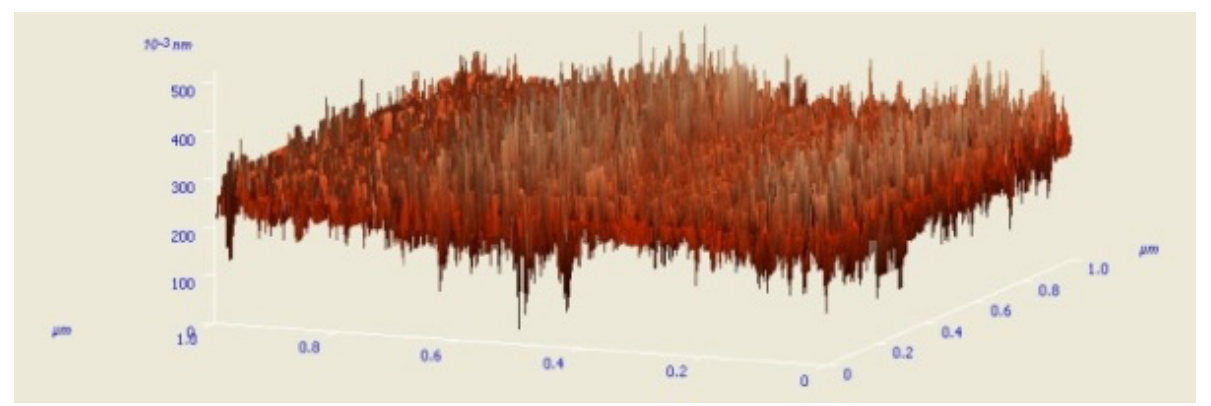

(a)

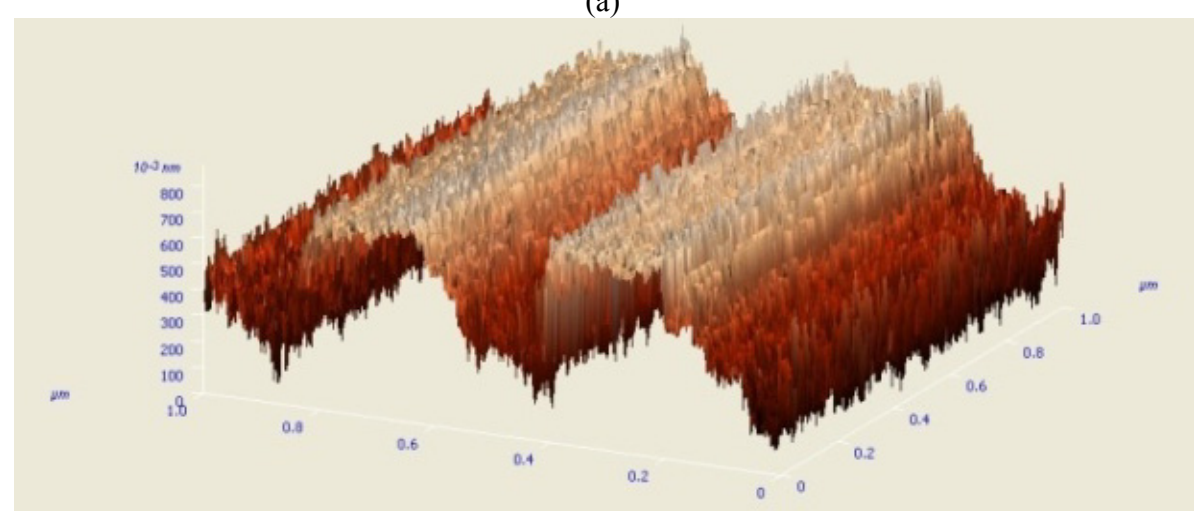

(b)

Fig. 6 (a) AFM picture of SiC sample pristine; (b) AFM picture of SiC irradiated to the spallation neutrons. 


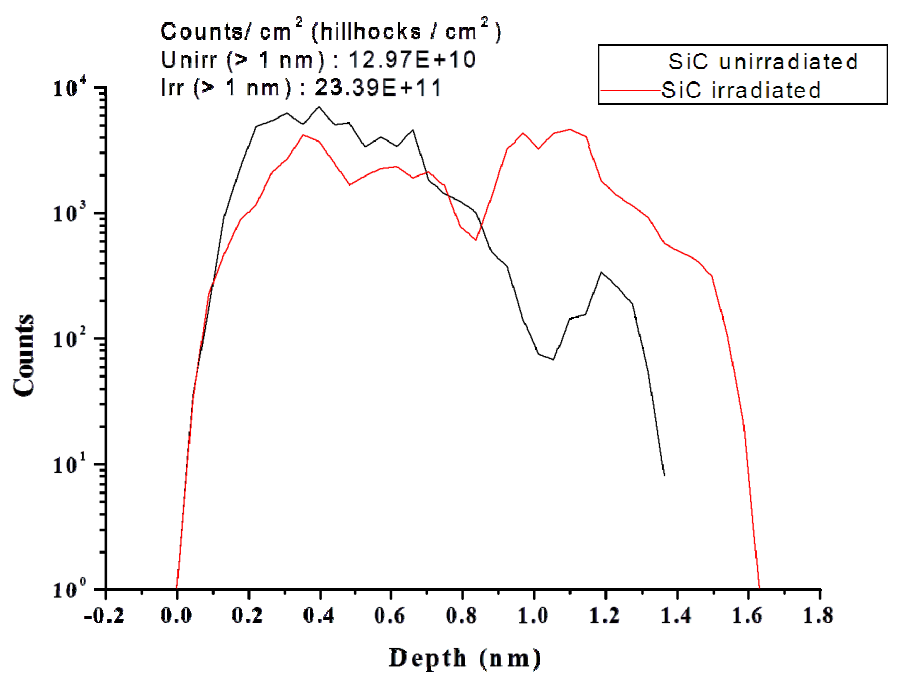

Fig. 7 Size distribution of hill-hocks in case of pristine and irradiated SiC sample.

Table 3 Results of Monte Carlo calculation of $E_{P K A}$ and DPA $\left(N_{d} /\right.$ atom/yr/n) calculated from $10^{10}$ histories of neutrons entering the $\mathrm{SiC}$ sample.

\begin{tabular}{lllll}
\hline$E_{n}(\mathrm{eV})$ & $n / \mathrm{cm}^{2} / \mathrm{s}$ & $N_{d} /(\mathrm{n} / p)$ & $E_{P K A}(\mathrm{eV})$ & $\mathrm{DPA} / \mathrm{yr}$ \\
\hline $4.85 \times 10^{3}$ & $2.65 \times 10^{4}$ & $1.4271 \times 10^{-2}$ & $1.04 \times 10^{3}$ & $7.83 \times 10^{-12}$ \\
$1.09 \times 10^{4}$ & $3.09 \times 10^{5}$ & $7.5862 \times 10^{-4}$ & $1.49 \times 10^{3}$ & $4.85 \times 10^{-12}$ \\
$1.64 \times 10^{4}$ & $6.36 \times 10^{5}$ & $8.8703 \times 10^{-4}$ & $3.09 \times 10^{3}$ & $1.17 \times 10^{-11}$ \\
$9.95 \times 10^{4}$ & $7.53 \times 10^{6}$ & $2.2968 \times 10^{-4}$ & $2.53 \times 10^{4}$ & $3.58 \times 10^{-11}$ \\
$1.95 \times 10^{6}$ & $4.89 \times 10^{6}$ & $2.0177 \times 10^{-2}$ & $3.02 \times 10^{5}$ & $2.05 \times 10^{-9}$ \\
$2.85 \times 10^{6}$ & $3.99 \times 10^{6}$ & $1.3653 \times 10^{-3}$ & $8.21 \times 10^{4}$ & $1.13 \times 10^{-10}$ \\
$4.18 \times 10^{6}$ & $2.19 \times 10^{6}$ & $2.1519 \times 10^{-1}$ & $7.51 \times 10^{5}$ & $9.77 \times 10^{-9}$ \\
$4.60 \times 10^{6}$ & $2.31 \times 10^{6}$ & $1.2258 \times 10^{-3}$ & $4.11 \times 10^{4}$ & $5.87 \times 10^{-11}$ \\
$5.06 \times 10^{6}$ & $2.32 \times 10^{6}$ & $2.2173 \times 10^{-2}$ & $8.20 \times 10^{5}$ & $1.07 \times 10^{-9}$ \\
\hline
\end{tabular}

\subsubsection{DPA}

Details of MC data $N_{d}$ for $\mathrm{SiC}$ in case of spallation neutrons is displayed in Table 3, from which average value of the $\mathrm{DPA} / \mathrm{yr} / n$ is deduced to be $1.44 \times 10^{-9}$ :

(1) From the results of $\mathrm{MC}$ simulation of $\mathrm{Ni}$ and $\mathrm{Nb}$ irradiated to spallation neutrons, it may be shown that, $\mathrm{DPA} / \mathrm{yr}$ is $3.18 \times 10^{-5}$ and $9.66 \times 10^{-6}$, respectively. They are $\sim 10^{3}$ to $10^{4}$ times higher than $1.44 \times 10^{-9}$ as obtained for the $\mathrm{SiC}$;

(2) In case of $\mathrm{AmBe}$ spectrum [7], DPA/yr for $\mathrm{Ni}$ and $\mathrm{Nb}$ comes out to be $1.8 \times 10^{-7}$ and $1.03 \times 10^{-7}$, respectively which is approximately 177 times smaller for the $\mathrm{Ni}$ and $~ 9.6$ times smaller in case of $\mathrm{Nb}$ compared to the values for the spallation neutron spectrum as given above in (1);

(3) $\mathrm{DPA} / \mathrm{yr}$ is plotted as a function of $E_{P K A}$ for the three samples in Fig. 8, and it shows that, DPA/yr/ $n$ is much smaller in case of $\mathrm{SiC}$ than $\mathrm{Nb}$ and $\mathrm{Ni}$.

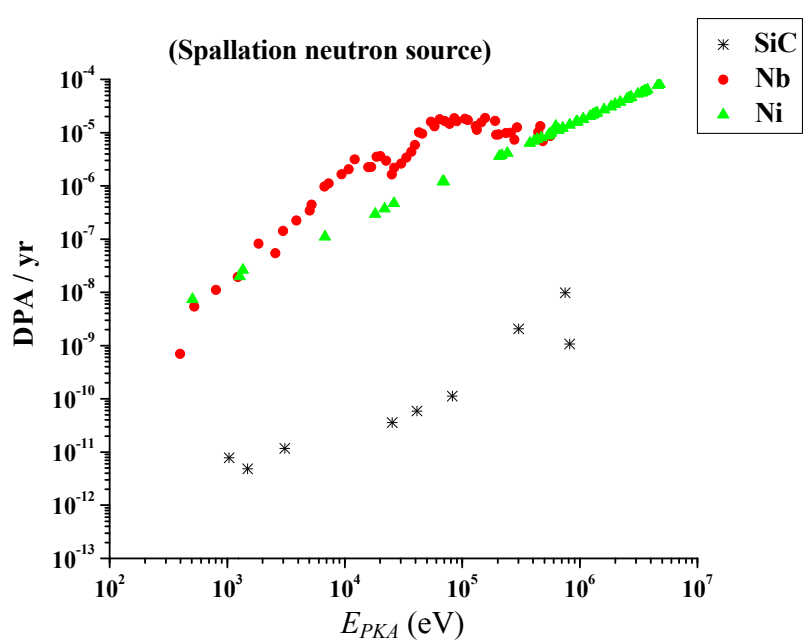

Fig. $8 \mathrm{DPA} / \mathrm{year} / \boldsymbol{n}$ versus $E_{P K A}(\mathrm{eV})$ in case of $\mathrm{SiC}, \mathrm{Nb}$ and $\mathrm{Ni}$ samples irradiated to the given spallation neutron spectrum. 


\section{Conclusions}

From the data of Monte Carlo simulations, following may be inferred:

(1) Defect production efficiency, $\eta$ as displayed in Fig. 4, is higher in case of metals than the SiC irradiated to the spallation neutrons. Evidently, effective threshold energy, $E_{d}$ eff $[7]$ is smaller in case of metals than the SiC. As a result, because DPA/yr/ $n$ depends on the Frenkel pairs, $N_{d}$, therefore, it is much smaller in case of $\mathrm{SiC}$ than the metals like $\mathrm{Ni}$ and $\mathrm{Nb}$;

(2) Based on $\mathrm{MC}$ results of $N_{d}$ and $T_{\text {dam }}$, a phenomenological relation is derived for understanding swelling coefficient, $\alpha_{s w}$. The coefficient is much smaller $(\sim 1 / 500)$ in case of $\mathrm{SiC}$ than Ni. Similar conclusion can be drawn from the observations that defect production efficiency, $\eta$ and DPA/yr are smaller in $\mathrm{SiC}$ than $\mathrm{Ni}$ or $\mathrm{Nb}$ metals. Conclusively, these observations can be interpreted in favor of $\mathrm{SiC}$ to be relatively highly radiation resistant towards the given spallation and AmBe neutron spectra;

(3) Based on the data of volume of the sample and values of " $k$ " given in Table 2 and using Eq. (14), swelling coefficient, $\alpha_{s w}$ for SiC irradiated to the given spectrum of spallation neutrons, comes out to be $\sim 8.53$ $\times 10^{-13} \mathrm{~cm}^{-1} \mathrm{eV}^{-1}$. In case of $\mathrm{Ni}$ metal after irradiation to a relatively softer neutron spectrum of AmBe source [7], $\alpha_{s w}$ has been estimated to be $4.7 \times 10^{-10} \mathrm{~cm}^{-1} \mathrm{eV}^{-1}$. This is about 500 times higher than $\mathrm{SiC}$ which is irradiated to a higher energy neutron spectrum. This may be interpreted in favor of $\mathrm{SiC}$ to be a radiation resistant material with respect to its swelling behavior. More experiments need to be performed in this direction for correlating swelling with the change in electrical and mechanical characteristics of the irradiated material.

\section{Acknowledgments}

Authors are thankful to DST (Department of Science and Technology) (India) for the grants under the ILTP (Integrated Long Term Program) on Science \& Technology Co-operation Number-A6.24 and
RFBR (Russian Foundation on Basic Research) project as well as to the JINR (Russia) for providing Phasatron beam.

\section{References}

[1] Bowman, C. D., Arthur, E. D., Lisowski, P. W., Lawrence, G. P., Jensen, R. J., Anderson, J. L., Blind, B., Cappiello, M., Davidson, J. W., England, T. R., Engel, L. N., Haight, R. C., Hughes III, H. G., Ireland, J. R., Krakowski, R. A., LaBauve, R. J., Letellier, B. C., Perry, R. T., Russell, G. J., Staudhammer, K. P., Versamis, G., and Wilson, W. B. 1992. "Nuclear Energy Generation and Waste Transmutation Using an Accelerator-Driven Intense Thermal Neutron Source." Nucl. Instrum. Methods A 320 (1-2): 336.

[2] Rubbia, C., Rubio, J. A., Buono, S., Carminati, F., Fietier, N., Galvez, J., Geles, C., Kadi, Y., Klapisch, R., Mandrillon, P., Revol, J. P., and Roche, C. 1995. "Conceptual Design of a Fast Neutron Operated High Power Energy Amplifier.” CERN/AT/95-44 (ET).

[3] Janssen, A. J. 2004. "Transmutation of Fission Products in Reactors and Accelerator-Driven Systems." ECN-R-94-001.

[4] Alexandar, R. 2008. "Radiation Damage Studies on Fusion Reactors." Presented at the IAEA Technical Meeting TM34567 on the Accelerator Simulation and Theoretical Modeling of Radiation Effects (SMoRE), Kharkov, Ukraine. Accessed November 16-19, 2009. http://www- naweb.iaea.org/napc/physics/meetings/ TM34567/login2.html.

[5] Broeders, C. H. M., and Konobeyev, A. Y. 2004. "Defect Production Efficiency in Metals under Neutron Irradiation." Journal of Nuclear Material 328 (2-3): 197-214.

[6] Greenwood, L. R. 1983. "A New Calculation of Thermal Neutron Damage and Helium Production in Nickel." $J$. Nucl. Mat. 115 (2-3): 137.

[7] Kumar, V., Raghaw, N. S., and Palsania, H. S. 2012. "A Monte Carlo Code for Radiation Damage by Neutrons." Nuclear Science and Engineering 172 (2): 151-63.

[8] “TALYS code." Accessed July 19, 2015. http://www. talys.eu.

[9] Broeders, C. H. M., Konobeyev, A. Y., and Voukelatou, K. 2004. IOTA-A Code to Study Ion Transport and Radiation Damage in Composite Materials. Karlsruhe: Forschungszentrum Karlsruhe, 1-2.

[10] Norgett, M. J., Robinson, M. T., and Torrens, I. M. 1975. "Standard Practice for Neutron Radiation Damage Simulation by Charged-Particle Irradiation." Annual Book of ASTM Standards. 